\title{
Catalytic control of plastic flow in siloxane-based liquid crys- talline elastomer networks
}

\author{
Mohand O. Saed, Eugene M. Terentjev \\ Cavendish Laboratory, University of Cambridge, Cambridge $\mathrm{CB}_{3}$ oHE, U. K.
}

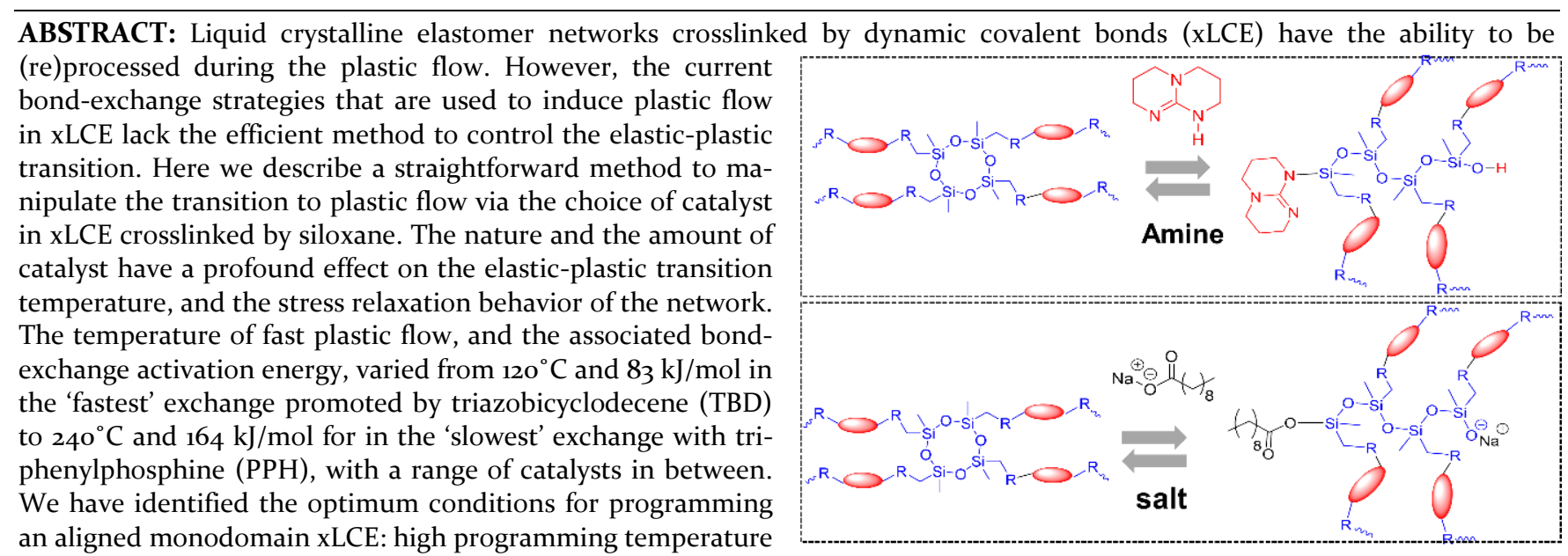
$\left(230^{\circ} \mathrm{C}\right)$ and low nematic to isotropic transition $\left(60^{\circ} \mathrm{C}\right)$, to achieve thermally and mechanically stable actuators.

Covalent crosslinking of liquid crystalline polymer chains forms a distinct reference state, with respect to which the changes in liquid crystalline order in elastomers can produce significant and reversible actuation up to $400 \%$ actuation strain., ${ }^{1,2}$ The large reversible actuation enables novel applications ranging from biomedical devices 3 to soft robotics. ${ }^{4}$ Establishing permanent molecular alignment (monodomain) in LCE is essential for load-free reversible actuation. The molecular alignment must be fixed by covalent crosslinking in the reference state, and can be achieved through one of the following three methods: mechanical strain with two-step crosslinking process, ${ }^{-7}$ surface alignment on a substrate, ${ }^{8,9}$ or crosslinking after shear extrusion. ${ }^{10,11}$ However, all of these methods can be difficult to use in practice (and in complex geometries) because the required molecular alignment must occur before final reaction is completed, which results in unavoidable competition between the alignment and the crosslinking.

Recently, vitrimers, or more broadly - polymer networks crosslinked by dynamic covalent bonds, emerge as a reliable technique to process polymer networks during the stressinduced plastic flow above a certain 'vitrification' temperature. $^{12,13}$ This term is used broadly, but it is well understood that this is not a transition temperature: the bond exchange is a thermally activated process, with its rate an exponential function of temperature, so there is a certain range of temperatures where the rate increases and the plastic flow becomes noticeable at the experimental time scale. This technique is utilized successfully in LCE to produce the 'ex- changeable' xLCE capable of re-alignment, recycling, and remolding, enabled by several dynamic covalent bonding chemistries. Transesterification ${ }^{14,15}$ was first employed as a bond-exchange mechanism to achieve complex alignment in xLCE, later followed by other strategies, such as disulfide, ${ }^{16}$ free-radical addition-fragmentation chain transfer, ${ }^{17,18}$ exchangeable urethane bonds, ${ }^{19}$ boronic transesterification, ${ }^{20}$ Diels-Alder dynamic bonds, ${ }^{21}$ and more recently siloxane bonds exchange ${ }^{22,23}$. However, all of these bond-exchange strategies lack the robust method to control the plastic flow above the nematic to isotropic transition temperature $\left(\mathrm{T}_{\mathrm{ni}}\right)$. Ideally, the plastic flow temperature (vitrification temperature, $T_{v}$ ) should be separated as far as possible from $T_{n i}$ so the useful actuation process is not affected by the plastic creep. In other words, $\mathrm{T}_{\mathrm{v}}$ should be relatively high to enable reprocessing, whereas $T_{n i}$ should be much lower to induce the thermal actuation in a mechanically stable network. In dynamic polymer networks, the rate of bond exchange increases rapidly with increasing temperature. ${ }^{12,14}$ If the activation energy of bond exchange is not very high, as a result of several thermal actuation cycles, the xLCE actuator would gradually lose its functionality due to the residual creep and the associated loss of molecular alignment. 


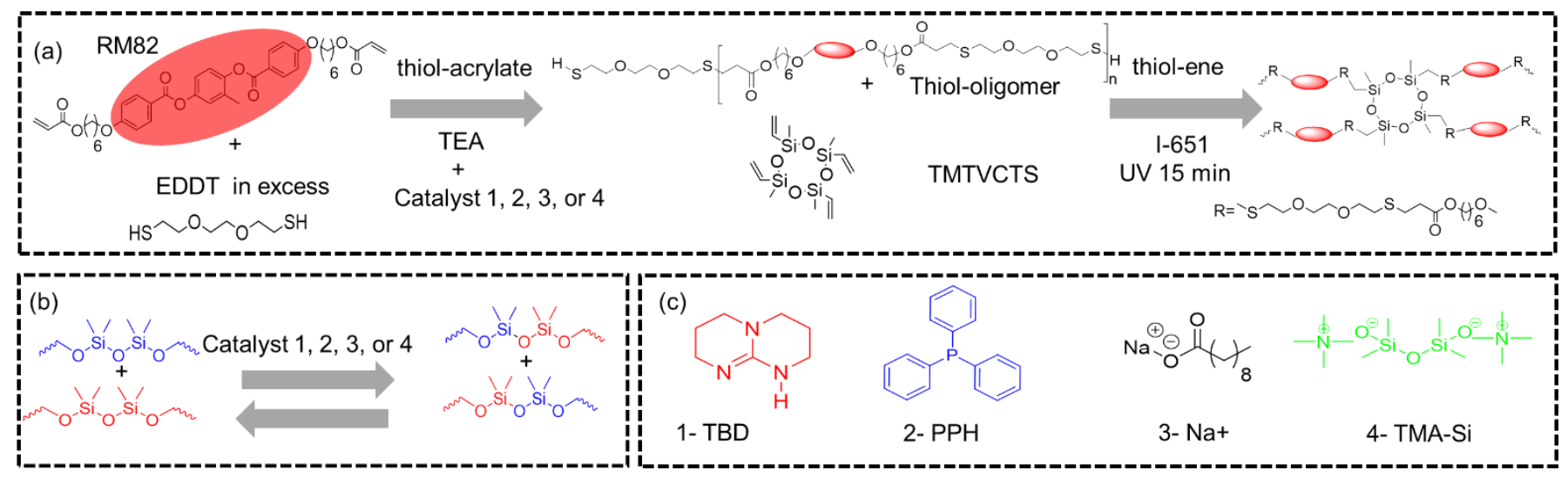

SCHEME 1. a) The summary of the reaction scheme: the mesogenic di-acrylate (RM82) first reacts with dithiol (EDDT), which is in excess. Secondly, the thiol-terminated oligomer chains are photo-polymerized with the vinyl bonds of the ring-siloxane to form a permanent (b) the general scheme of siloxane exchange enabled by base catalyst. (c) Catalysts used to enable the siloxane bonds exchange

In this paper, we introduce a systematic method to control the plastic flow transition via the choice of catalyst using siloxane-based liquid crystalline networks. Siloxane chemistries are ideal to produce high-performance LCE due to their superior thermomechanical properties (low glass transition, high failure strain, and high actuation stroke), compared to the traditional hydrocarbon materials. ${ }^{24}$ The equilibrium exchange of siloxane dynamic bonds has been known since 1954 when Osthoff et al. discovered that bond exchange can be triggered via acid or base catalyst at an elevated temperature. ${ }^{25,26}$ This interesting discovery has long been overlooked, because the polymer community at the time was interested in developing stable and "permanent" networks. In 2012 McCarthy et al. showed that this powerful siloxane bond exchange can be used to reprocess crosslinked polydimethylsiloxane (PDMS) post polymerization. ${ }^{27}$ However, the thermal stability (e.g. plastic flow induced by bonds exchange) of this class of dynamic networks remains largely unexplored even in isotropic siloxane elastomers.

Controlling the elastic-plastic transition temperature is very important, as it largely dictates the processability and applications of the dynamically crosslinked networks. For instance, processing thermally active materials such as xLCE requires high plastic flow temperature. Moderate plastic flow temperature can be attractive for recycling and remolding of regular siloxane networks such as PDMS. Low plastic flow temperature is useful for self-healing applications, where the activation of the siloxane bonds needs to be around room temperature. Here we study chemical methods to control this transition.

Generally, the thermal instability (activation of siloxane bonds) is dictated by the nature and the amount of catalyst, and the crosslinking density of the network. For example, increasing the amount and degree of basicity of the catalyst lowers the activation temperature of the siloxane bonds (and thus lowers the plastic flow temperature). A number of studies have shown that a small amount of mild base (e.g. o.1 to $0.2 \mathrm{wt} \%$ of bis(tetramethylammonium) oligodimethylsiloxanediolate) allows for rapid bond exchange occurring from 90 to $140^{\circ} \mathrm{C} .{ }^{27,28}$ This temperature range is controlled by the crosslinking density, with higher density (shorter network strands) resulting in the higher plastic flow transition temperature; this is a suitable range to recycle and remold the ordinary PDMS. ${ }^{27}$ Whereas stronger base of quaternary ammonium salt (e.g. tetramethylammonium hydroxide) enables siloxane bond exchange at much lower temperatures ranging from 5 to $60^{\circ} \mathrm{C} .29$ Low temperature siloxane bonds exchange is successfully utilized to produce self-healing PDMS. Likewise, an appropriate amount of aggressive base (e.g. primary or secondary amine) can completely solubilize crosslinked siloxane networks due to the fast reversible siloxane-amine exchange at room temperature. $3^{\circ}$ This may have the potential to chemically recycle PDMS at relativity mild temperatures. Building upon these studies, here we describe a systematic method to control the plastic flow via catalyst in siloxane based xLCE.

It is important to note that we are using the same crosslinking density, keeping this variable of the process fixed: we are only changing the nature and the amount of catalyst. We are guided by the recent work on using the thiolacrylate/ene reaction to design LCE with uniform and highly controllable network structures.4,22,31 We rationally incorporated exchangeable siloxane bonds within the LCE networks to enable the plastic flow at distinct temperatures in the

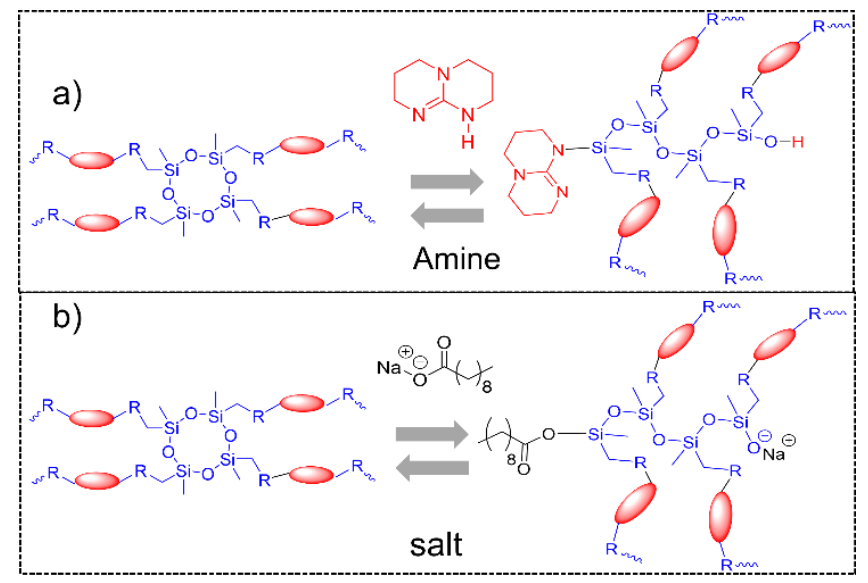

SCHEME 2. The principal route of siloxane exchange in our network via nucleophilic cleavage of Si-O bonds via (a) base catalyst or (b) salts. 
presence of a catalyst. We first prepare LC oligomer chains via the self-limiting thiol-acrylate Michael addition between mesogenic diacrylate, RM82 (at 1 molar ratio) and isotropic dithiol, EDDT (in excess, at 1.4 molar ratio). The Michael addition is catalyzed via an amine-based catalyst TEA, unless otherwise indicated. The di-thiol oligomer is then radically crosslinked with a 0.4 molar ratio vinyl siloxane crosslinker, TMTVCTS (Scheme 1a). The overall reaction scheme is similar to a previously reported method. ${ }^{22,31,32}$ To systematically investigate the influence of catalyst, we selected a wide range of catalytic systems varying from strong base (triazobicyclodecene, TBD), nucleophilic agent (triphenylphosphine, $\mathrm{PPH}$ ), mild base salts (sodium octanoate, $\mathrm{Na}^{+}$), and tetramethylammonium siloxanolate, TMA-Si), see Scheme 1c for chemical structures. In general, the mechanisms of catalysis of these systems have been well-studied since the 1950 and attributed to nucleophilic cleavage of $\mathrm{Si}-\mathrm{O}$ bonds (Scheme 1b). ${ }^{25,26,33}$ However, the mechanism of catalysis by TBD is different from those of metal or siloxanate salts. TBD allows for reversible bonds exchange between crosslinker bonds ( $\mathrm{Si}$ $\mathrm{O})$ and TBD (N-H) bonds (scheme 2a)..$^{\circ}$ The other catalysts enable generation of reactive end group capable of anionic

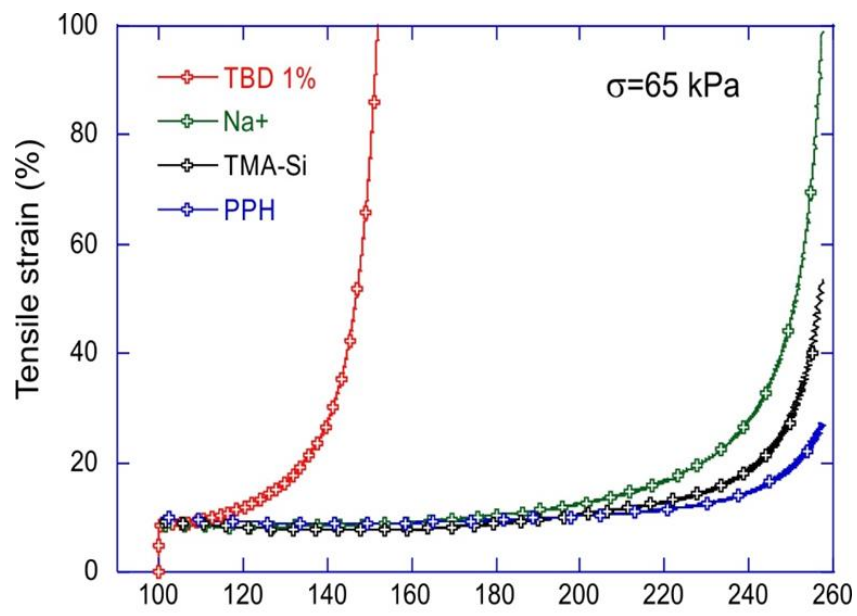

(a)

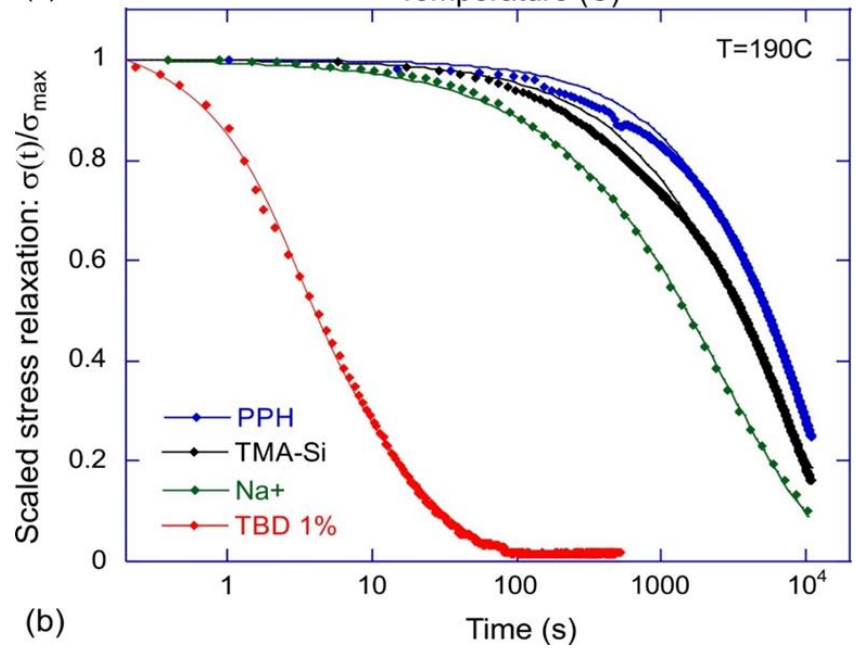

Figure 1. (a) Iso-force temperature ramp plots for all catalysts at the same $1 \%$, using constant engineering stress of $65 \mathrm{kPa}$ and heating rate of $2 \%$ min. (b) Normalized stress realaxtion as a founction of time at $190^{\circ} \mathrm{C}$ for the same materials, data fitted by the exponential relaxation model. polymerization (scheme $\mathbf{2 b}$ )..$^{27,33}$ Variation in catalysis mechanism, reactivity, and their own thermal stability produces dynamic networks with very different plastic flow temperature, stress relaxation, and bond-exchange activation energy.

It is important to identify the decomposition temperature $\left(\mathrm{T}_{\mathrm{d}}\right)$ of a network crosslinked by dynamic covalent bonds to find the processing temperature window (the processing of such material must take place well below $T_{d}$ ). Thermogravimetric analysis (TGA) is initially used to study the decomposition of each xLCE network containing 1 wt $\%$ of a catalyst under nitrogen (Figure Sia, Supporting information). Most of the examined polymer networks show remarkable thermal stability, where the $5 \%$ mass loss occurs above $300{ }^{\circ} \mathrm{C}$ (TMA$\mathrm{Si}, \mathrm{Na}+$, and $\mathrm{PPH})$. On the other hand, the sample which contains $1 \mathrm{wt} \% \mathrm{TBD}$ exhibits significantly lower $\mathrm{T}_{\mathrm{d}}\left(270^{\circ} \mathrm{C}\right)$ compared to the other catalysts. This can be attributed to the unique mechanism of TBD catalysis. The interaction between TBD and the siloxane crosslinker generates significant number hydroxy groups, (-OH-) (Scheme 2a). These hydroxy groups can result in side reactions with the ester groups (COO-) in the mesogen. However, the tendency of this side reaction decreases as $T_{d}$ increases with decreasing the amount of TBD (which we test and illustrate in Figure Sıb, Supporting information). Having established the decomposition limit for our materials, we processed to test thermal properties for our xLCE networks at temperatures at least $50^{\circ}$ $C$ below $\mathrm{T}_{\mathrm{d}}$.

Constant force ('iso-force') temperature ramp experiment is an efficient method to characterize the elastic-plastic transition 34,35 (Figure 1a). This experiment monitors the extensional strain in the elastic (permanent siloxane bonds) and the plastic (exchanging siloxane bonds) regions, at constant tensile stress at increasing temperature. In a permanent rubbery network, at constant stress, one expects the strain to decrease, as the rubber modulus increases linearly with temperature (entropic elasticity). Transition to plastic flow is then marked by a change in the slope of this curve, as the magnitude strain starts increasing with temperature in the plastic region - this temperature of onset of plastic regime is listed for all materials in Table S1, Supporting information. At a much higher temperature, the strain finally diverges when the rapid flow regime sets in. This point depends on the rate of heating, but broadly defines the upper boundary of the elastic-plastioc transition region, also listed in Table S1, Supporting information. This experiment can be a little complicated in an xLCE material35 due to the effect of thermal actuation below $\mathrm{T}_{\text {ni }}$, therefore, we start at $\mathrm{T}=100^{\circ} \mathrm{C}$ : well in the isotropic phase (see the DSC plot, Figure S2, Supporting information). The plastic flow temperature range for TBD is much lower compared to other catalysts, all using equal amount of catalyst at $1 \mathrm{wt} \%$. This can be attributed to the difference in the catalysis mechanism, since TBD uses its reactive secondary amine bond to form reversible $\mathrm{Si}-\mathrm{O}$ bonds. $3^{\circ}$ As expected, the stress relaxation follows the same trend as observed in the iso-force test, confirming that TBD relaxes the stress much faster at $190^{\circ} \mathrm{C}$ (Figure 1b). $\mathrm{PPH}$, TMA-Si, and $\mathrm{Na}^{+}$have fairly similar plastic flow and stress relaxation behavior, due to the similarity in their catalysis mechanism. 
The effect of the amount of catalyst has been studied in a variety of dynamic covalent bond systems using both theoretical and experimental models. 34 Most of the studies suggested that the exchange reaction rate varies with the catalyst concentration (until saturation at a high concentration). Therefore, we investigated the influence of TBD concentration on both plastic flow and stress relaxation, varying the TBD concentrations from 1, 0.5, 0.2, and o.1 wt\% (Figure 2). Increasing the concentration of TBD leads to a decrease in the plastic flow temperature (Figure $3 \mathbf{a}$ ) and an increase in the stresses relaxation rate (Figure $\mathbf{3} \mathbf{b}$ ). This can be attributed to the diffusion rate of catalyst (increasing the probability of meeting the reacting groups, see 'rate of attempts' below). It is important to note, we were not able to reach catalytic saturation in our system. Beyond the saturation concentration any increase in catalytic concentration will not further affect the plastic flow temperature and the stress relaxation. This is because the reaction systems we adapted (thiol-ene radical polymerization) prevent us from using amine catalyst at higher than $5 \mathrm{~mol} \%$ (Scheme 1 ), as a recent study from the

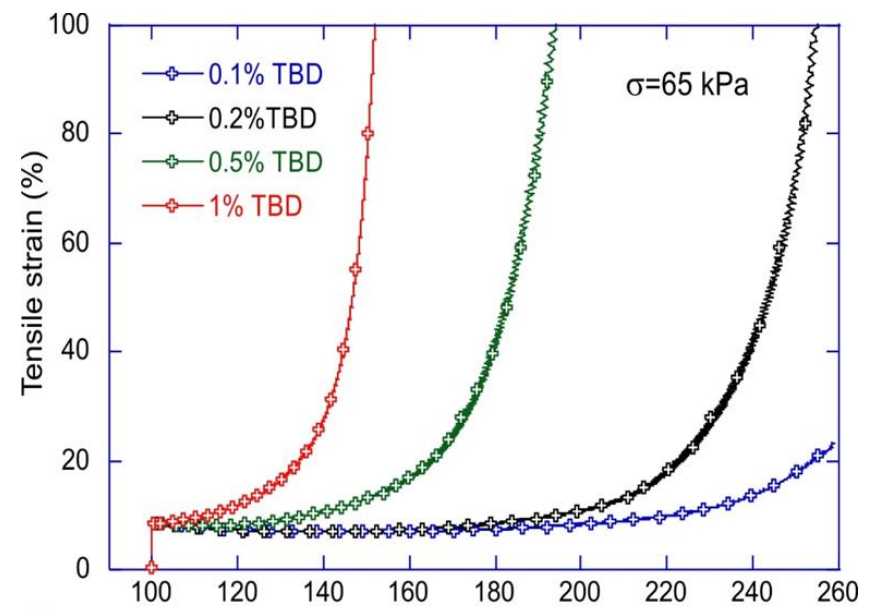

(a) Temperature $(\mathrm{C})$

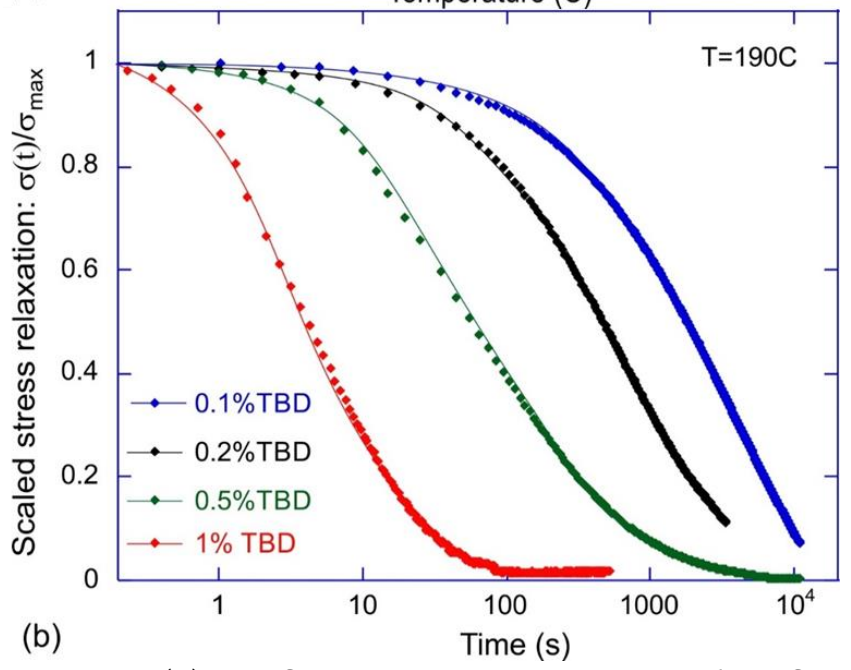

Figure 2. (a) Iso-force temperature ramp plots for four different concentration of TBD, using constant engineering stress of $65 \mathrm{kPa}$ and heating rate of $2^{\circ} / \mathrm{min}$. (b) Normalized stress realaxtion as a founction of time at $190^{\circ} \mathrm{C}$ for the same TBD concentrations, data fitted by the exponential relaxation model.
Bowman group suggested that excessive amine adversely affected the thiol-ene reaction conversation. ${ }^{36}$ Therefore we used amine concentration below that threshold. Furthermore, using larger amount of TBD can produce significant amount of free hydroxy group upon its interaction with the siloxane groups which may initiate unwanted transesterification reaction with ester groups in the mesogenic monomer.

To further investigate the details of the bond-exchange reaction, and the influence of catalyst (presenting TBD, Na, TMA-Si, and PPH), we studied the stress relaxation of our xLCE networks at different temperatures. This is a classical experiment, illustrated in Figure za for PPH: after a fast step in strain, the relaxing stress is scaled and fitted by a model exponential relaxation curve $\bar{\sigma}(t)=e^{-t / \tau}$. The relaxation time $\tau$, obtained by such a fitting is a strong function of temperature: relaxation is much faster at higher temperatures, according to the activation law: $\tau(T)=\tau_{0} e^{\Delta G / k_{B} T}$, where $\Delta G$ is the free energy of reaction activation and $\tau_{0}^{-1}$ is called the 'rate of attempts' (determined by the diffusion of catalyst and the accessibility of the reaction site). Figure $\mathbf{3} \mathbf{b}$ presents the
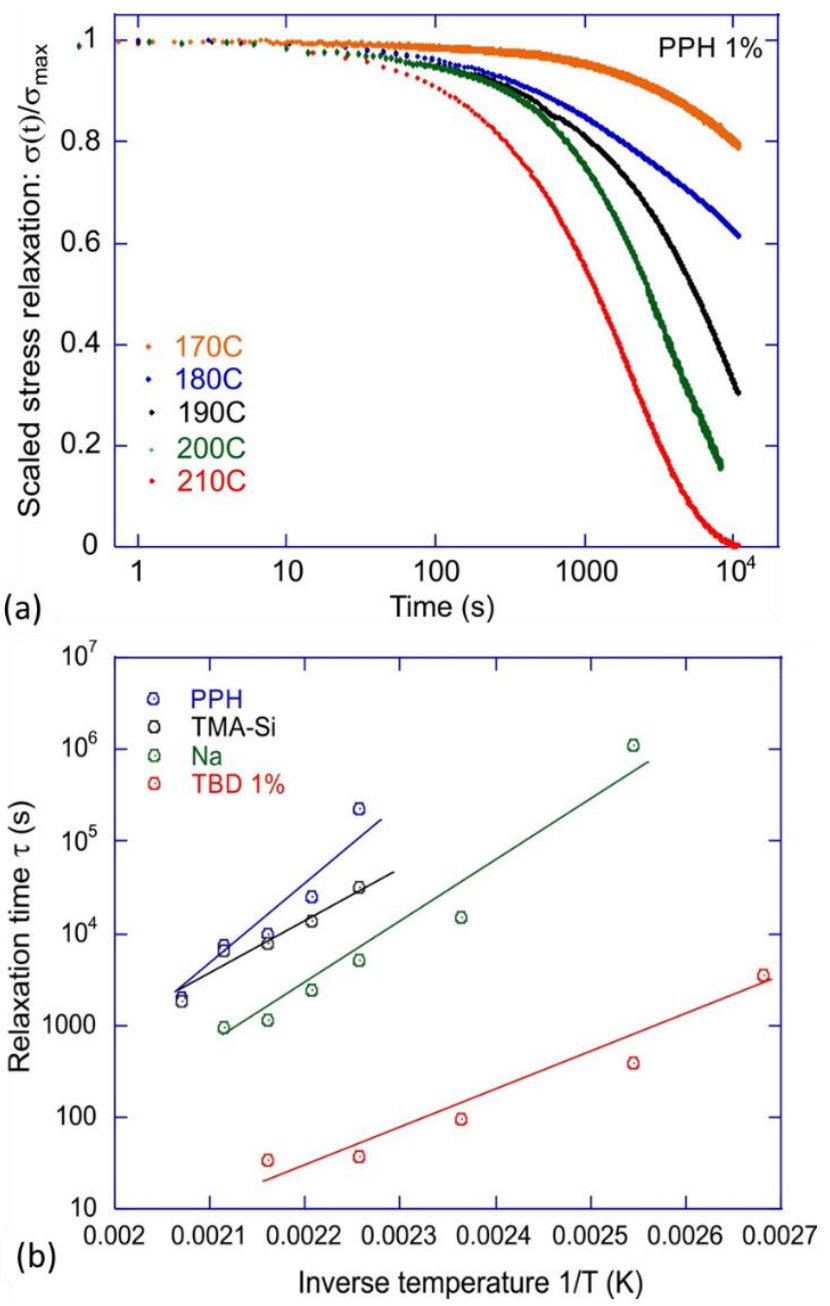

Figure 3. (a) Normalized stress realaxtion plots for 1wt\% PHH, at several temperaures. (b) Arrhenius plots of the stress relaxation time $\tau(\mathrm{T})$ for all catalysts (at $1 \mathrm{wt} \%)$. The linear fitting of $\ln (\tau)$ vs. $1 / \mathrm{T}$ gives the activation energy of the bond exchange. 
results of many relaxation experiments summarized in the Arrhenius plot, showing the $\ln (\tau)$ vs. $1 / T$. The slope of the fitted line gives the value of activation energy $\Delta G$ while the vertical elevation of each data set indicates the changing rate of attempts. In the increasing order, we obtained the activation energy TBD $(83 \mathrm{~kJ} / \mathrm{mol})$, compared to TMA-Si (116 $\mathrm{kJ} / \mathrm{col}), \mathrm{Na}+(128 \mathrm{~kJ} / \mathrm{mol})$, and finally $\mathrm{PHH}(164 \mathrm{~kJ} / \mathrm{mol})$. On the other hand, comparing the data for TBD at different concentrations, we found that the activation energy of the reaction is the same, while the observed difference in relaxation time is due to the difference in the rate of attempts: the vertical offset in the Arrhenius plot (see Table S2 and Figure S4, Supporting information). On reflection, this is reassuring, since the reaction mechanism (and so the activation energy) are indeed the same.

To have an LCE material perform as an actuator, its internal alignment needs to be fixed in a certain pattern (in the simplest case - as a uniform aligned monodomain). xLCEs are typically aligned (programmed) during the plastic flow where bonds reshuffling occurs rapidly. Applying constant stress to xLCEs isothermally at a temperate above the range of elastic-plastic transition can result in a large creep. Our previous study shows that $100 \%$ strain (creep induced) is sufficient to affect permanent uniform alignment in these materials. The nature and amount of catalyst not only alter the plastic flow temperature $\left(\mathrm{T}_{\mathrm{v}}\right)$ and the stress relaxation
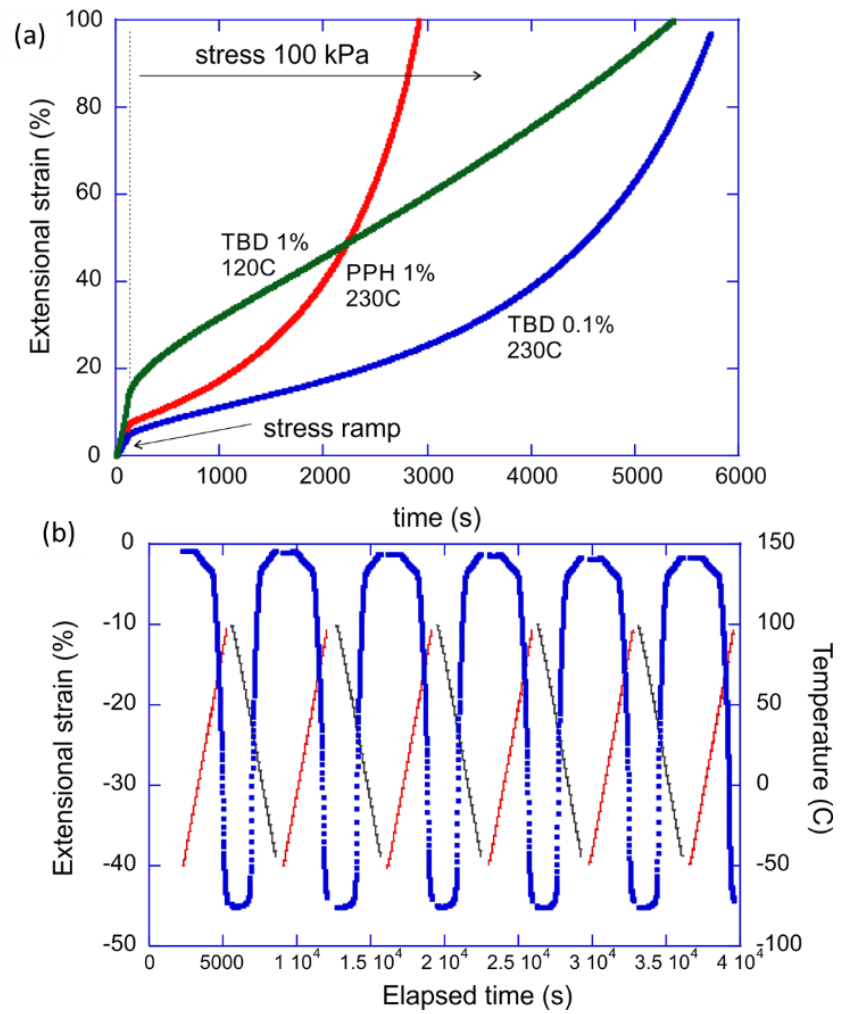

Figure 4. a) Unixal alignment of monodomain in xLCE. Programing temperaure depends on nature and concentration of catalyst. Sample can be programed at $120{ }^{\circ} \mathrm{C}$ for $1 \mathrm{wt} \%$ TBD or $230{ }^{\circ} \mathrm{C}$ for 0.1 wt $\%$ TBD or 1 wt $\%$ PPH. b) Thermal acuation over 5 heating cycles for 1 wt\% PPH. The sample is thermaly cycled between -50 to $100^{\circ} \mathrm{C}$ behavior, but also influence the optimal temperature at which xLCE samples can be programed. To demonstrate the effect of the catalyst on the programing temperature, we compared the effect of TBD and PPH (using the equal amount of $1 \mathrm{wt} \%$ each). These catalysts are selected because of their non-volatility (high boiling temperatures: $263^{\circ} \mathrm{C}$ for TBD and $377^{\circ} \mathrm{C}$ for $\mathrm{PPH}$ ) and their ability to allow programming at low or high temperature, owing to their very different rates of bond exchange. It is important to note that the amount of TBD can also be varied from 1 to $0.1 \mathrm{wt} \%$, which also enables programming xLCEs at low or high temperatures, respectively. The programming temperature must be selected within the plastic flow region (above the $T_{v}$ and below $\mathrm{T}_{\mathrm{d}}$ ). Here, we chosen $120^{\circ} \mathrm{C}$ as an ideal programming temperature for sample containing $1 \mathrm{wt} \%$ of TBD, and $230^{\circ} \mathrm{C}$ for samples containing $1 \mathrm{wt} \% \mathrm{PPH}$, and similarly for $0.1 \mathrm{wt} \%$ TBD. The effect of catalyst on programming is illustrated in Figure 4a. To achieve a creep of $100 \%$ strain, the engineering stress is quickly ramped to $100 \mathrm{kPa}$, isothermally at $120^{\circ} \mathrm{C}$ for 1 wt $\% \mathrm{TBD}$, or at $230^{\circ} \mathrm{C}$ for $0.1 \mathrm{wt} \% \mathrm{TBD}$ or 1 wt $\% \mathrm{PPH}$. The creep time is strongly affected by the applied stress; hence one may choose different conditions depending on whether the fast or slow programming is required. Sample with $1 \mathrm{wt} \% \mathrm{PPH}$ reaches the target $100 \%$ strain twice as fast than $0.1 \mathrm{wt} \%$ TBD (and the rest of the samples, not shown in the plot), even though PPH has the largest bond-exchange activation energy $\Delta G$. This result suggests that the creep time at constant stress can also be influenced by the nature of catalyst, and the corresponding rate of attempts of the reaction. The contrasting result for $1 w t \%$ TBD in Figure $4 \mathbf{a}$ is because we chose a much lower temperature for this programming process: this illustrates how to influence the programming process even for a catalyst with the highest rate of bond exchange.

As we have mentioned in the introduction, it is best to design xLCE actuators with a high programing temperature to enable re-programing, and a low $\mathrm{T}_{\text {ni }}$ to induce thermal actuation in the mechanically stable regime. Since $T_{n i}$ does not change with changing the nature or the amount of catalyst $\left(-60^{\circ} \mathrm{C}\right.$ for all samples regardless of their type), therefore, samples with $1 \mathrm{wt} \% \mathrm{PPH}$ or $0.1 \mathrm{wt}$ TBD are ideal candidates to achieve thermally stable actuators, due to the large separation between the programing temperature $\left(230^{\circ} \mathrm{C}\right)$ and $\mathrm{T}_{\mathrm{ni}}$ $\left(60^{\circ} \mathrm{C}\right)$. Thermal actuation cycles for xLCE with $1 \mathrm{wt} \% \mathrm{PPH}$ are shown in Figure $\mathbf{4} \mathbf{b}$. The sample is cycled between -50 and $100^{\circ} \mathrm{C}$ with a constant rate of $3 \mathrm{~K} / \mathrm{min}$. The magnitude of actuation under small bias stress of $10 \mathrm{kPa}$ is $\sim 45 \%$ strain. As expected, the sample maintains stable actuation over several heating and cooling cycles without a creep decay. The same stable actuation pattern is found for xLCE with o.1 wt\% strain (Figure $\mathrm{S}_{4}$, Supporting information).

In conclusion, we report the design of xLCE materials based on siloxane bonds exchange using the click reactions (thiol-acrylate and thiol-ene). We have shown that siloxane bond exchange can be tuned by altering the nature and the amount of catalyst. The nature and the amount of catalyst have a profound effect on the elastic-plastic transition temperature, the stress relaxation, and the monodomain programing temperature of the LCE networks. As a result, we were able to design mechanically and thermally stable xLCE actuators. 
Supporting Information is available free of charge via the Internet at http://pubs.acs.org.

Experimental and characterization details: Materials and preparation method, Thermal gravimetric analysis (TGA), Differential Scanning Calorimetry (DSC), and additional Stress Relaxation and Actuation Measurements.

\section{AUTHOR INFORMATION}

\section{Corresponding Author}

* Eugene M. Terentjev emt1ooo@cam.ac.uk

\section{Author Contributions}

The work was carried out, the data analyzed, and the manuscript written through contributions of all authors.

\section{Funding Sources}

This work was funded by the European Research Council Hzo2o Advanced Grant 786659.

\section{ACKNOWLEDGMENT}

The authors are grateful to Alexandra Gablier and Yan Ji for help and useful discussions.

\section{REFERENCES}

(1) Warner, M.; Terentjev, E. M. Liquid Crystal Elastomers; Oxford university press, 2007; (OUP, Oxford)

(2) Tajbakhsh, A. R.; Terentjev, E. M. Spontaneous Thermal Expansion of Nematic Elastomers. Eur. Phys. J. E 2001, 6 (2), 181-188.

(3) Volpe, R. H.; Mistry, D.; Patel, V. V.; Patel, R. R.; Yakacki, C. M. Dynamically Crystalizing LiquidCrystal Elastomers for an Expandable EndplateConforming Interbody Fusion Cage. Adv. Healthc. Mater. 2020, 9 (1), 1901136.

(4) Kotikian, A.; McMahan, C.; Davidson, E. C.; Muhammad, J. M.; Weeks, R. D.; Daraio, C.; Lewis, J. A. Untethered Soft Robotic Matter with Passive Control of Shape Morphing and Propulsion. Sci. Robot. 2019, 4 (33), 7044.

(5) Küpfer, J.; Finkelmann, H. Nematic Liquid Single Crystal Elastomers. Makromol. Chemie, Rapid Commun. 1991, 12 (12), 717-726.

(6) Küpfer, J.; Finkelmann, H. Liquid Crystal Elastomers: Influence of the Orientational Distribution of the Crosslinks on the Phase Behaviour and Reorientation Processes. Macromol. Chem. Phys. 1994, 195 (4), 13531367.

(7) Yakacki, C. M.; Saed, M.; Nair, D. P.; Gong, T.; Reed, S. M.; Bowman, C. N. Tailorable and Programmable Liquid-Crystalline Elastomers Using a Two-Stage Thiol-Acrylate Reaction. RSC Adv. 2015, 5 (25),

18997-19001.

(8)

Broer, D. J.; Boven, J.; Mol, G. N.; Challa, G. In-situ Photopolymerization of Oriented Liquid-crystalline Acrylates, 3. Oriented Polymer Networks from a Mesogenic Diacrylate. Macromol. Chem. Phys. 1989, 190 (9), 2255-2268.

(9) Ware, T. H.; McConney, M. E.; Wie, J. J.; Tondiglia, V. P.; White, T. J. Voxelated Liquid Crystal Elastomers. Science. 2015, 347 (6225), 982-984.

(10) Ahir, S. V; Tajbakhsh, A. R.; Terentjev, E. M. Selfassembled Shape-memory Fibers of Triblock Liquidcrystal Polymers. Adv. Funct. Mater. 2006, 16 (4), 556-56o.

(11) Ambulo, C. P.; Burroughs, J. J.; Boothby, J. M.; Kim, H.; Shankar, M. R.; Ware, T. H. Four-Dimensional Printing of Liquid Crystal Elastomers. ACS Appl. Mater. Interfaces 2017, 9 (42), 37332-37339.

(12) Montarnal, D.; Capelot, M.; Tournilhac, F.; Leibler, L. Silica-like Malleable Materials from Permanent Organic Networks. Science. 2011, 334 (6058), 965968.

(13) Kloxin, C. J.; Bowman, C. N. Covalent Adaptable Networks: Smart, Reconfigurable and Responsive Network Systems. Chem. Soc. Rev. 2013. 42 (17), 71617173 .

(14) Pei, Z.; Yang, Y.; Chen, Q.; Terentjev, E. M.; Wei, Y.; Ji, Y. Mouldable Liquid-Crystalline Elastomer Actuators with Exchangeable Covalent Bonds. Nat. Mater. 2014, 13 (1), 36-14.

(15) Hanzon, D. W.; Traugutt, N. A.; McBride, M. K.; Bowman, C. N.; Yakacki, C. M.; Yu, K. Adaptable Liquid Crystal Elastomers with TransesterificationBased Bond Exchange Reactions. Soft Matter 2018, 14 (6), 951-96o.

(16) Li, Y.; Zhang, Y.; Rios, O.; Keum, J. K.; Kessler, M. R. Photo-Responsive Liquid Crystalline Epoxy Networks with Exchangeable Disulfide Bonds. RSC Adv. 2017, 7 (59), 37248-37254.

(17) McBride, M. K.; Martinez, A. M.; Cox, L.; Alim, M.; Childress, K.; Beiswinger, M.; Podgorski, M.; Worrell, B. T.; Killgore, J.; Bowman, C. N. A Readily Programmable, Fully Reversible Shape-Switching Material. Sci. Adv. 2018, 4 (8), eaat4634.

(18) Davidson, E. C.; Kotikian, A.; Li, S.; Aizenberg, J.; Lewis, J. A. 3D Printable and Reconfigurable Liquid Crystal Elastomers with Light-Induced Shape Memory via Dynamic Bond Exchange. Adv. Mater. 2020. 32 (1), 1905682.

(19) Wen, Z.; McBride, M. K.; Zhang, X.; Han, X.; Martinez, A. M.; Shao, R.; Zhu, C.; Visvanathan, R.; Clark, N. A.; Wang, Y. Reconfigurable LC Elastomers: Using a Thermally Programmable Monodomain to 
Access Two-Way Free-Standing Multiple Shape Memory Polymers. Macromolecules 2018, 51 (15), 5812-5819.

(20) Saed, M. O.; Gablier, A.; Terentejv, E. M. Liquid Crystalline Vitrimers with Full or Partial BoronicEster Bond Exchange. Adv. Funct. Mater. 2019. 30 (3), 201906458.

(21) Jiang, Z.; Xiao, Y.; Yin, L.; Han, L.; Zhao, Y. "SelfLockable" Liquid-Crystalline Diels-Alder Dynamic Network Actuators with Room Temperature Programmability and Solution-Reprocessability. Angew. Chemie Int. Ed. 2020. 59 (12), 4925-4931.

(22) Saed, M. O.; Terentjev, E. M. Siloxane Crosslinks with Dynamic Bond Exchange Enable Shape Programming in Liquid-Crystalline Elastomers. Sci. Reports. 2020. 10 (1), 6609.

(23) Wu, Y.; Yang, Y.; Qian, X.; Chen, Q.; Wei, Y.; Ji, Y. Liquid-Crystalline Soft Actuators with Switchable Thermal Reprogrammability. Angew. Chemie - Int. Ed. 2020. 59 (12), 4778-4784.

(24) Finkelmann, H.; Kock, H.; Rehage, G. Investigations on Liquid Crystalline Polysiloxanes 3. Liquid Crystalline Elastomers-a New Type of Liquid Crystalline Material. Makromol. Chemie, Rapid Commun. 1981, 2 (4), 317-322.

(25) Kantor, S. W.; Grubb, W. T.; Osthoff, R. C. The Mechanism of the Acid-and Base-Catalyzed Equilibration of Siloxanes. J. Am. Chem. Soc. 1954, 76 (20), 5190-5197.

(26) Osthoff, R. C.; Bueche, A. M.; Grubb, W. T. Chemical Stress-Relaxation of Polydimethylsiloxane Elastomersi. J. Am. Chem. Soc. 1954, 76 (18), 46594663 .

(27) Zheng, P.; McCarthy, T. J. A Surprise from 1954: Siloxane Equilibration Is a Simple, Robust, and Obvious Polymer Self-Healing Mechanism. J. Am. Chem. Soc. 2012, 134 (4), 2024-2027.

(28) Moskalenko, Y. E.; Bagutski, V.; Thiele, C. M. Chemically Synthesized and Cross-Linked PDMS as Versatile Alignment Medium for Organic Compounds. Chem. Commun. 2017, 53 (1), 95-98.

(29) Schmolke, W.; Perner, N.; Seiffert, S. Dynamically Cross-Linked Polydimethylsiloxane Networks with Ambienterature Self-Healing. Macromolecules 2015, 48 (24), 8781-8788.

(30) Hsiao, Y. -C; Hill, L. W.; Pappas, S. P. Reversible Amine Solubilization of Cured Siloxane Polymers. J. Appl. Polym. Sci. 1975, 19 (10), 2817-2820.

(31) Saed, M. O.; Ambulo, C. P.; Kim, H.; De, R.; Raval, V.; Searles, K.; Siddiqui, D. A.; Cue, J. M. O.; Stefan, M. C.; Shankar, M. R. Molecularly-Engineered, 4DPrinted Liquid Crystal Elastomer Actuators. Adv.
Funct. Mater. 2019, 29 (3), 1806412.

(32) Zheng, N.; Hou, J.; Zhao, H.; Wu, J.; Luo, Y.; Bai, H.; Rogers, J. A.; Zhao, Q.; Xie, T. Mechano-Plastic Pyrolysis of Dynamic Covalent Polymer Network toward Hierarchical $3 \mathrm{D}$ Ceramics. Adv. Mater. 2019, 31 (11), 1807326.

(33) Gilbert, A. R.; Kantor, S. W. Transient Catalysts for the Polymerization of Organosiloxanes. J. Polym. Sci. 1959, 40 (136), 35-58.

(34) Capelot, M.; Unterlass, M. M.; Tournilhac, F.; Leibler, L. Catalytic Control of the Vitrimer Glass Transition. ACS Macro Lett. 2012. 1 (7),783-792

(35) Pritchard, R. H.; Redmann, A.-L.; Pei, Z.; Ji, Y.; Terentjev, E. M. Vitrification and Plastic Flow in Transient Elastomer Networks. Polymer. 2016, 95, 45-51.

(36) Love, D.; Fairbanks, B.; Bowman, C. Reaction Environment Effect on the Kinetics of Radical ThiolEne Polymerizations in the Presence of Amines and Thiolate Anions. ACS Macro Lett. 2020. 9 (2), 174-179 
